\title{
Particle Trajectories at a Magnetic Neutral Point
}

\author{
Kaname Amano and Takao TsudA \\ Department of Electrical Engineering, Hokkaido University, \\ Sapporo, Japan
}

(Received May 2, 1977; Revised November 12, 1977)

\begin{abstract}
The motion of charged particles is studied numerically by a test-particle approach. A Petschek-type electromagnetic field is given for a plasma in which a finite electric conductivity, necessary at a magnetic neutral point, results from the inertia of the particles. The plasma is assumed to be cold in the external flow region. It is found that two gyration frequencies characterize the motions parallel and perpendicular to the external magnetic field. The meandering electrons are concentrated near the neutral point and have a mean velocity, though with a considerable dispersion, big enough to cause some current-induced instabilities.
\end{abstract}

\section{Introduction}

The reconnection of magnetic field lines at a neutral point is important in understanding astrophysical and geophysical phenomena such as solar flares and magnetospheric substorms. It was first proposed by DUNGEY (1953) as a possible source of high energy particles, then developed by Parker (1963), Petschek (1964), Sonnerup (1970), and YeH and Axford (1970). These studies are based on MHD (magnetohydrodynamic) analyses and reviewed critically by VASYliUnas (1975). However, the neutral point is sometimes collisionless in the sense that the flight time of charged particles in the diffusion region is much smaller than the mean collision time. The particle trajectories are important near the neutral point.

In this paper, we investigate the motion of charged particles by a testparticle approach. A Petschek-type electromagnetic field is given for a plasma in which the finite electric conductivity, necessary at the neutral point, results from the inertia of the particles. The plasma is assumed to be cold in the external flow region. Adiabatic theory cannot be used and a numerial computation must be made. It is found that two gyration frequencies characterize the motions parallel and perpendicular to the external magnetic field. We look into some properties of the meandering electrons which are concentrated near the neutral point.

Particle trajectories in the simplified model current sheets, which corre- 
spond to the field reversal region outside the neutral region, have been studied by Speiser $(1965,1968)$, Sonnerup (1971), and EAStwood (1972). It is too complicated to solve the field configuration and the particle motion self-consistently (AMANo and TSUDA, 1977); therefore, the single particle approach is worth trying to gain physical insight.

\section{A Petschek-Type Field Geometry}

It is shown by VASYliunas (1975) that the reconnection model proposed by Petscher (1964) is valid also for a plasma in which the finite electric conductivity results from the inertia of charged particles. As shown by SPEISER (1970), the life time of particles in a system can replace the longer collision time in the expression for the conductivity. Namely, the life time is the flight time of particles in the diffusion region and is construed as the traverse time of fluid in MHD descriptions.

Figure 1 shows a Petschek-type field geometry for the reconnection. It is two-dimensional in the sense that there is no magnetic field in the $z$-direction and every quantity depends only on $x$ and $y$, but not on $z$. The slow waves are standing along the separatrix surfaces between the external flow region and the field reversal region. The half thickness of the field reversal region $X$ is given by

$$
\begin{aligned}
X / x^{*} & =\left(1+y^{2} / y^{* 2}\right)^{1 / 2}, \\
x^{*} & =c / \omega_{\mathrm{pe}}, \quad \omega_{\mathrm{pe}}=\left(n e^{2} / \varepsilon_{0} m_{\mathrm{e}}\right)^{1 / 2}, \\
y^{*} & =x^{*} / M_{\mathrm{A}}, \\
M_{\mathrm{A}} & =U_{0} / V_{\mathrm{A}}, \quad V_{\mathrm{A}}=B_{0} /\left(\mu_{0} n\left(m_{\mathrm{e}}+m_{\mathrm{p}}\right)\right)^{1 / 2},
\end{aligned}
$$

where $M_{\mathrm{A}}$ is the Alfvén Mach number defined as the ratio of the plasma flow

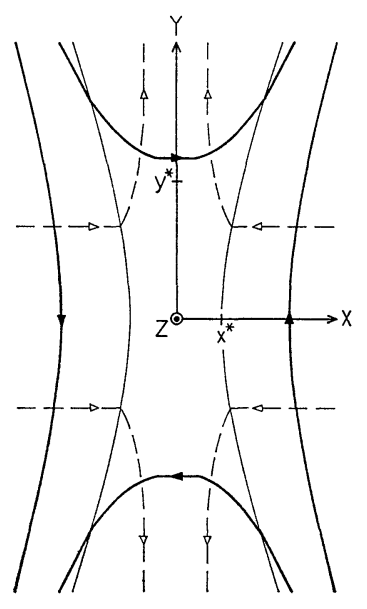

Fig. 1. Petschek-type field geometry for the reconnection. Solid and broken lines are for the magnetic field and the plasma flow, respectively. The neutral point is at the origin of the coordinate system. 
velocity $U_{0}$ to the Alftén velocity $V_{\mathrm{A}}$ in the external flow region. This Alfvén Mach number is proportional to the merging rate of the magnetic field and called the reconnection rate. The other quantities $\omega_{\mathrm{pe}}, n, c, m_{\mathrm{e}}, m_{\mathrm{p}}, e, \varepsilon_{0}$ and $\mu_{0}$ are the electron plasma frequency, plasma density, light velocity, electron mass, proton mass, electron charge, dielectric constant and magnetic permeability, respectively. The neutral point is at the origin of the coordinate system. The magnetic field decays owing to the finite conductivity in the diffusion region $|x|<x^{*}$ and $|y|<y^{*}$, whereas it is frozen in the plasma of the surrounding regions.

The magnetic field $\boldsymbol{B}=\left(B_{x}, B_{y}, 0\right)$ is given by

$$
\begin{aligned}
& B_{x}=M_{\mathrm{A}} B_{0}\left(3\left(\frac{y^{2} / y^{* 2}}{1+y^{2} / y^{* 2}}\right)^{1 / 2}-\left(\frac{y^{2} / y^{* 2}}{1+y^{2} / y^{* 2}}\right)^{3 / 2}\right) \operatorname{sign}(y), \\
& B_{y}=B_{0} \operatorname{sign}(x)
\end{aligned}
$$

in the external flow region $|x|>X$, and

$$
\begin{aligned}
B_{x} & =M_{\mathrm{A}} B_{0}\left(2\left(\frac{y^{2} / y^{* 2}}{1+y^{2} / y^{* 2}}\right)^{1 / 2}-\left(\frac{y^{2} / y^{* 2}}{1+y^{2} / y^{* 2}}\right)^{3 / 2}\right) \operatorname{sign}(y), \\
B_{y} & =0, \quad \text { if }|x|<X-x^{*}, \\
& =B_{0}\left(|x| / x^{*}-\left(X / x^{*}-1\right)\right) \operatorname{sign}(x), \quad \text { if } \quad X-x^{*}<|x|,
\end{aligned}
$$

in the field reversal region $|x|<X$. Equation (2) can be obtained approximately from the conservation of the field component normal to the wave front. We assume a linear profile of $B_{y}$ across the discontinuity of the width $x^{*}$. The electric field $\boldsymbol{E}=\left(0,0, E_{z}\right)$ is uniform in a steady state and given by

$$
E_{z}=M_{\mathrm{A}} V_{\mathrm{A}} B_{0} .
$$

The above situation can exist for $M_{\mathrm{A}} \lesssim 0.1$.

3. Particle Trajectories at the Neutral Point

The equation of motion

$$
\mathrm{d} \boldsymbol{v} / \mathrm{d} t=q / m(\boldsymbol{E}+\boldsymbol{v} \times \boldsymbol{B})
$$

for a particle of mass $m$, charge $q$ and velocity $\boldsymbol{v}=\left(v_{x}, v_{y}, v_{z}\right)$ is written as

$$
\begin{gathered}
\mathrm{d} v_{x} / \mathrm{d} t=-v_{z} \Omega_{y}, \\
\mathrm{~d} v_{y} / \mathrm{d} t=v_{z} \Omega_{x}, \\
\mathrm{~d} v_{z} / \mathrm{d} t=V_{\mathrm{A}} \Omega_{x 0}+v_{x} \Omega_{y}-v_{y} \Omega_{x}, \\
\boldsymbol{\Omega}=q \boldsymbol{B} / m, \quad \Omega_{x 0}=M_{\mathrm{A}} \Omega_{0}, \quad \Omega_{0}=q B_{0} / m,
\end{gathered}
$$

in terms of its components. We numerically integrate Eqs. (6)-(8) with the 
initial position $\boldsymbol{r}_{0}=\left(x_{0}, y_{0}, z_{0}\right)$ and the initial velocity $\boldsymbol{v}_{0}=\left(v_{x 0}, v_{y 0}, v_{z 0}\right)$ in the electromagnetic field given by Eqs. (2)-(4). The gyration frequencies $\Omega_{x}$ and $\Omega_{y}$ are associated with the motions parallel (to be exact, in the $y-z$ plane) and perpendicular (in the $x-z$ plane) to the $y$-axis, respectively. Equation (7) and the $-v_{y} \Omega_{x}$ term in Eq. (8) give the parallel motion. Equation (6) and the $v_{x} \Omega_{y}$ term in Eq. (8) give the perpendicular motion. Note that $B_{x} \approx M_{\mathrm{A}} B_{0}, B_{y} \approx B_{0}$ and so $\Omega_{x} / \Omega_{y} \approx M_{\mathrm{A}} \ll 1$ except near the $y$-axis.

Figures 2(a)-(e) show the typical motion of electrons in the Petschek-type field geometry for the case $M_{\mathrm{A}}=0.05$ : temporal variations of the velocity com-

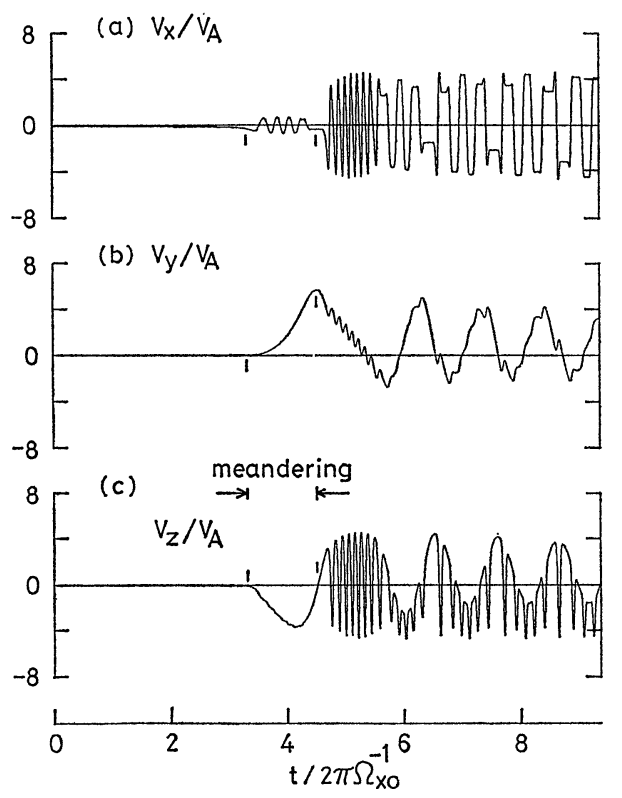

(d)

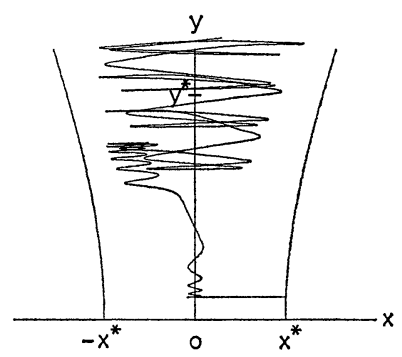

(e)

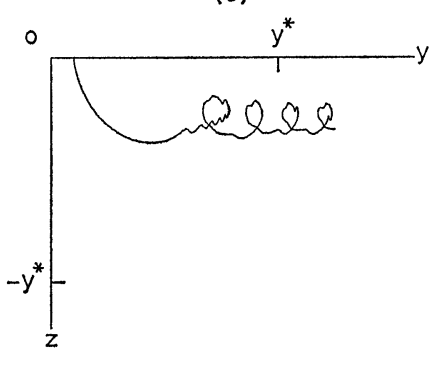

Fig. 2. Typical motion of the electron in the Petschek-type field geometry for the case $M_{\mathrm{A}}=0.05$ : temporal variations of the velocity components (a) $v_{x}$, (b) $v_{y}$ and (c) $v_{z}$, and the trajectories in the (d) $x-y$ and (e) $y-z$ planes, respectively. The neutral point is indicated by 0 . 
ponents (a) $v_{x}$, (b) $v_{y}$ and (c) $v_{z}$, and the trajectories in the (d) $x-y$ and (e) $y-z$ planes, respectively. The initial conditions are

$$
\boldsymbol{r}_{0}=\left(X\left(0.1 y^{*}\right), 0.1 y^{*}, 0\right), \quad \boldsymbol{v}_{0}=\left(-M_{\mathrm{A}} V_{\mathrm{A}}, 0,0\right) .
$$

Figures $3(a)-(e)$ show the typical motion of protons for the same conditions. Note that time is scaled by $2 \pi / \Omega_{x 0 \mathrm{e}}$ and $2 \pi / \Omega_{x 0 \mathrm{p}}$ in Figs. 2 and 3 , respectively.

The charged particles coming into the diffusion region perpendicularly to the external magnetic field experience three distinct phases. The first phase is char-
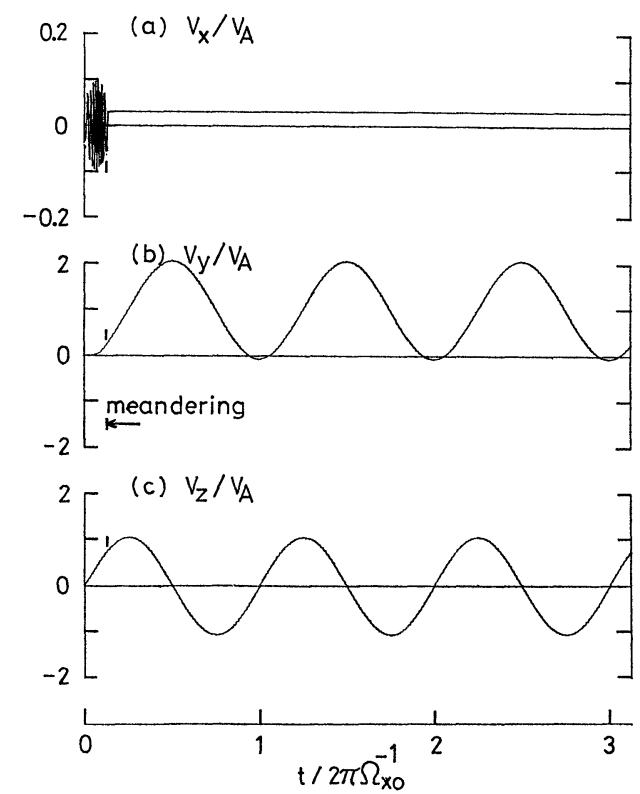

(d)

(e)
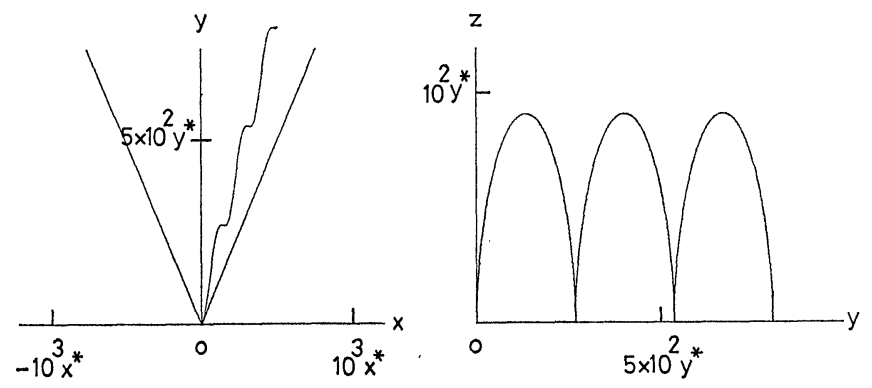

Fig. 3. Typical motion of the proton in the Petschek-type field geometry for the case $M_{\mathrm{A}}=0.05$ : temporal variations of the velocity components (a) $v_{x}$, (b) $v_{y}$ and (c) $v_{z}$, and the trajectories in the (d) $x-y$ and (e) $y-z$ planes, respectively. The neutral point is indicated by 0 . 
acterized by the $\boldsymbol{E} \times \boldsymbol{B}$ drift into the diffusion region perpendicular to the $y$-axis. For $|x| \geq X-x^{*}$, the equation of motion in the $x-z$ plane is approximated by

$$
\begin{aligned}
& \mathrm{d} v_{x} / \mathrm{d} t=-v_{z} \Omega_{y}, \\
& \mathrm{~d} v_{z} / \mathrm{d} t=M_{\mathrm{A}} V_{\mathrm{A}} \Omega_{0}+v_{x} \Omega_{y},
\end{aligned}
$$

since $\Omega_{x} \ll \Omega_{y}$. Forces depend on the velocity $M_{\mathrm{A}} V_{\mathrm{A}}$ and the gyration frequency $\Omega_{y}$. In the external flow region $|x|>X$ where $\Omega_{y}=\Omega_{0}$, the trajectory is a trochoid

$$
\begin{aligned}
& -x+\left(x_{0}-\frac{M_{\mathrm{A}} V_{\mathrm{A}}}{\Omega_{0}}\left(\frac{v_{z 0}}{M_{\mathrm{A}} V_{\mathrm{A}}}-\theta\right)\right)=\frac{M_{\mathrm{A}} V_{\mathrm{A}}}{\Omega_{0}}\left(\Omega_{0} t+\theta-\frac{Q}{M_{\mathrm{A}} V_{\mathrm{A}}} \sin \left(\Omega_{0} t+\theta\right)\right), \\
& z-\left(z_{0}+\frac{M_{\mathrm{A}} V_{\mathrm{A}}}{\Omega_{0}}\left(\frac{v_{x 0}}{M_{\mathrm{A}} V_{\mathrm{A}}}\right)\right)=\frac{M_{\mathrm{A}} V_{\mathrm{A}}}{\Omega_{0}}\left(1-\frac{Q}{M_{\mathrm{A}} V_{\mathrm{A}}} \cos \left(\Omega_{0} t+\theta\right)\right), \\
& \sin \theta=v_{z 0} / Q, \quad \cos \theta=\left(M_{\mathrm{A}} V_{\mathrm{A}}+v_{x 0}\right) / Q, \quad Q=\left(\left(M_{\mathrm{A}} V_{\mathrm{A}}+v_{x 0}\right)^{2}+v_{z 0}^{2}\right)^{1 / 2},
\end{aligned}
$$

here the initial position is taken to be $x_{0}>X$. If $\left|v_{x 0}\right| \ll M_{\mathrm{A}} V_{\mathrm{A}}$ and $\left|v_{z 0}\right| \ll M_{\mathrm{A}} V_{\mathrm{A}}$, it becomes a cycloid

$$
\begin{gathered}
-x+x_{0}-\frac{v_{z 0}}{\Omega_{0}}=\frac{M_{\mathrm{A}} V_{\mathrm{A}}}{\Omega_{0}}\left(\Omega_{0} t-\sin \Omega_{0} t\right), \\
z-z_{0}-\frac{v_{x 0}}{\Omega_{0}}=\frac{M_{\mathrm{A}} V_{\mathrm{A}}}{\Omega_{0}}\left(1-\cos \Omega_{0} t\right) .
\end{gathered}
$$

The pitch lengths for the electron and the proton are respectively given by

$$
\begin{aligned}
& \Delta x_{\mathrm{e}}=2 \pi M_{\mathrm{A}} V_{\mathrm{A}} / \Omega_{0 \mathrm{e}} \approx 2 \pi M_{\mathrm{A}}\left(m_{\mathrm{e}} / m_{\mathrm{p}}\right)^{1 / 2} x^{*} \ll x^{*}, \\
& \Delta x_{\mathrm{p}}=2 \pi M_{\mathrm{A}} V_{\mathrm{A}} / \Omega_{0 \mathrm{p}} \approx 2 \pi M_{\mathrm{A}}\left(m_{\mathrm{p}} / m_{\mathrm{e}}\right)^{1 / 2} x^{*},
\end{aligned}
$$

and $\Delta x_{\mathrm{p}}<x^{*}$ if $M_{\mathrm{A}}<1 / 2 \pi\left(m_{\mathrm{p}} / m_{\mathrm{e}}\right)^{1 / 2}=3.7 \times 10^{-3}$. The gyration radius defined as $M_{\mathrm{A}} V_{\mathrm{A}} / \Omega_{0}$ becomes $r_{\mathrm{g} 0}=\Delta x / 2 \pi$.

When the particle comes in the discontinuity $X-x^{*}<|x|<X$, a $\nabla_{\perp} B$ drift, in the positive (negative) $z$-direction for an electron (proton), adds to the $\boldsymbol{E} \times \boldsymbol{B}$ drift toward the $y$-axis. The $\nabla_{\perp} B$ drift velocity is given by $\boldsymbol{v}_{\nabla_{\perp} B}=\mu \boldsymbol{B} \times \nabla_{\perp} B / q B^{2}$. The magnetic moment is defined as $\mu=-m v_{\perp}^{2} \boldsymbol{B} / 2 B^{2}$. Here, $\bar{\nabla}_{\perp}$ and $v_{\perp}$ are the differential operator and the velocity component perpendicular to $\boldsymbol{B}$, respectively. The ratio of the drift velocities for a particle with $v_{\perp}=M_{\mathrm{A}} V_{\mathrm{A}}$ is approximated by $v_{\nabla_{\perp} B} / v_{E \times B}=r_{\mathrm{g} 0} / 2 x^{*}=\Delta x / 4 \pi x^{*}$. Therefore, the $\nabla_{\perp} B$ drift has a minor effect if the plasma is cold in the external flow region.

The second phase is characterized by the meandering motion that consists of a drift in the $y-z$ plane leaving the neutral point and an uncoupled oscillation perpendicular to the $y$-axis. In the diffusion region, the particle can be accelerated by the electric field not restricted by the strong magnetic field. The electron (proton) is accelerated first in the negative (positive) $z$-direction as $v_{z} \approx$ $V_{\mathrm{A}} \Omega_{x 0} t$ from Eq. (8), then parallel to the $y$-axis leaving the neutral point as 
$\mathrm{d} v_{y} / \mathrm{d} t \approx V_{\mathrm{A}} \Omega_{x 0} \Omega_{x} t$ from Eq. (7). As long as $v_{z}$ is negative (positive), Eq. 6 implies an oscillation in $x$ perpendicular to the $y$-axis, since it becomes $\mathrm{d}^{2} x / \mathrm{d} t^{2}=$ $-k x$ where approximately $k=v_{z} \Omega_{0} / x^{*}>0$. The oscillation becomes damped or growing according as $k$ increases or decreases. As suggested by SPEISER (1965, 1968) and EASTwood (1972), the term $v_{x} \Omega_{y}$ in Eq. (8) can be neglected as it adds only a small oscillation. The equation of motion in the $y-z$ plane is approximated by

$$
\begin{aligned}
& \mathrm{d} v_{y} / \mathrm{d} t=v_{z} \Omega_{x}, \\
& \mathrm{~d} v_{z} / \mathrm{d} t=V_{\mathrm{A}} \Omega_{x 0}-v_{y} \Omega_{x},
\end{aligned}
$$

which are associated with $V_{\mathrm{A}}$ and $\Omega_{x}$. It describes the motion of the guiding center. Note that Eq. (15) does not depend on $M_{\mathrm{A}}$ if the time and length are scaled by $1 / \Omega_{x 0}$ and $y^{*}$, respectively.

Thus the magnetic field component $B_{y}$, which is negligible in the diffusion region by PetscheK (1964), has not a significant influence on the ejection of plasma from the neutral region. It is expected that, for a low- $\beta$ plasma, the initial velocity components $v_{x 0}$ and $v_{z 0}$ have a major effect on the uncoupled oscillation and a minor effect on the motion in the $y-z$ plane. The condition for a particle with the perpendicular velocity $M_{\mathrm{A}} V_{\mathrm{A}}$ to be trapped within the field reversal region is roughly given by $r_{\mathrm{g} 0}<x^{*}$. After $v_{z}$ changes its sign, $x$ will no longer oscillate.

The third phase is characterized by the $\boldsymbol{E} \times \boldsymbol{B}$ drift parallel to the $y$-axis away from the neutral point, and sometimes also by a coupled oscillation perpendicular to the $y$-axis. Inside the discontinuities $|x|<X-x^{*}$, the motion of charged particles is given by Eq. (15), where $\Omega_{x} \approx 2 \Omega_{x 0} y / y^{*}$ for $y<0.5 y^{*}$ and $\Omega_{x} \approx \Omega_{x 0}$ for $y>0.5 y^{*}$. When $\Omega_{x}=\Omega_{x 0}$, the trajectory is a trochoid

$$
\begin{aligned}
& y-\left(y_{0}+\frac{V_{\mathrm{A}}}{\Omega_{x 0}}\left(\frac{v_{z 0}}{V_{\mathrm{A}}}-\theta\right)\right)=\frac{V_{\mathrm{A}}}{\Omega_{x 0}}\left(\Omega_{x 0} t+\theta-\frac{Q}{V_{\mathrm{A}}} \sin \left(\Omega_{x 0} t+\theta\right)\right), \\
& z-\left(z_{0}-\frac{V_{\mathrm{A}}}{\Omega_{x 0}}\left(\frac{v_{y 0}}{V_{\mathrm{A}}}\right)\right)=\frac{V_{\mathrm{A}}}{\Omega_{x 0}}\left(1-\frac{Q}{V_{\mathrm{A}}} \cos \left(\Omega_{x 0} t+\theta\right)\right), \\
& \sin \theta=v_{z 0} / Q, \quad \cos \theta=\left(V_{\mathrm{A}}-v_{y 0}\right) / Q, \quad Q=\left(\left(V_{\mathrm{A}}-v_{y 0}\right)^{2}+v_{z 0}^{2}\right)^{1 / 2} .
\end{aligned}
$$

If $\left|v_{y 0}\right| \ll V_{\mathrm{A}}$ and $\left|v_{z 0}\right| \ll V_{\mathrm{A}}$, it becomes a cycloid

$$
\begin{aligned}
& y-y_{0}-\frac{v_{z 0}}{\Omega_{x 0}}=\frac{V_{\mathrm{A}}}{\Omega_{x 0}}\left(\Omega_{x 0} t-\sin \Omega_{x 0} t\right), \\
& z-z_{0}+\frac{v_{y 0}}{\Omega_{x 0}}=\frac{V_{\mathrm{A}}}{\Omega_{x 0}}\left(1-\cos \Omega_{x 0} t\right) .
\end{aligned}
$$

The pitch lengths for the electron and the proton are respectively given by 


$$
\begin{aligned}
& \Delta y_{\mathrm{e}}=2 \pi V_{\mathrm{A}} / \Omega_{x 0 \mathrm{e}} \approx 2 \pi\left(m_{\mathrm{e}} / m_{\mathrm{p}}\right)^{1 / 2} y^{*} \ll y^{*}, \\
& \Delta y_{\mathrm{p}}=2 \pi V_{\mathrm{A}} / \Omega_{x 0 \mathrm{p}} \approx 2 \pi\left(m_{\mathrm{p}} / m_{\mathrm{e}}\right)^{1 / 2} y^{*} \gg y^{*} .
\end{aligned}
$$

The gyration radius defined as $V_{\mathrm{A}} / \Omega_{x 0}$ becomes $r_{\mathrm{g} x}=\Delta y / 2 \pi$.

For the electron of small mass, $v_{z}$ changes its sign from negative to positive and the uncoupled oscillation stops nearly at $y=0.5 y^{*}$. Till then, it is accelerated along the meandering orbit to a higher velocity than the Alfvén velocity. The trajectory becomes a trochoid in the $y-z$ plane for $y>0.5^{*}$, with which the oscillation perpendicular to the $y$-axis is coupled. Equation (8) is written as $\mathrm{d} v_{z} / \mathrm{d} t=\left(V_{\mathrm{A}} \Omega_{x 0}-v_{y} \Omega_{x}\right)+v_{x} \Omega_{y}$. Note that $V_{\mathrm{A}} \Omega_{x 0}-v_{y} \Omega_{x}$ is a slowly varing term
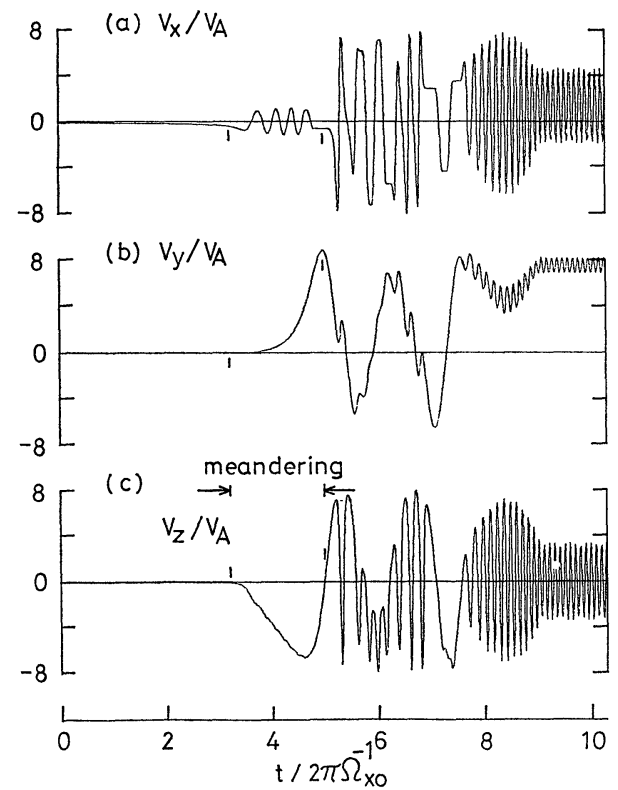

(d)

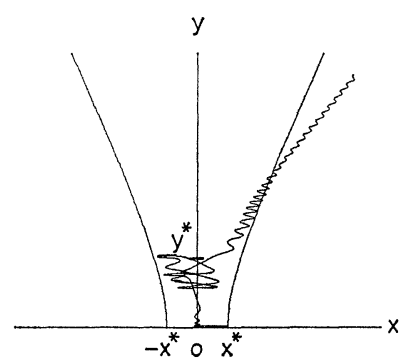

(e)

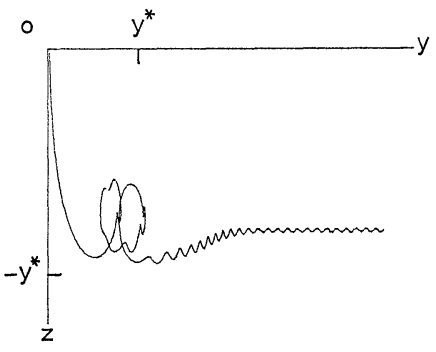

Fig. 4. Motion of an electron for the case $M_{\mathrm{A}}=0.1$ and $y_{0}=0.02 y^{*}$ : temporal variations of the velocity components (a) $v_{x}$, (b) $v_{y}$ and (c) $v_{z}$, and the trajectories in the (d) $x-y$ and (e) $y-z$ planes, respectively. 
characterized by $\Omega_{x}$ and approximately becomes $\left(V_{\mathrm{A}}-v_{y}\right) \Omega_{x 0}$ for $y>0.5 y^{*}$. During $V_{\mathrm{A}} \Omega_{x 0}-v_{y} \Omega_{x}>0\left(\Omega_{x 0}<0\right.$ for electron $)$ or $v_{y}>V_{\mathrm{A}}$ for $y>0.5^{*}$, therefore, Eqs. (6) and (8) may cause a drift toward the external flow region accompanied with a transition from a meandering to a gyration orbit as shown in Fig. 2(d). An inverse transition is also possible. A few electrons actually may go out of the field reversal region along the magnetic field lines.

Figures 4(a)-(e) show an example for the case $M_{\mathrm{A}}=0.1$ : temporal variations of the velocity components (a) $v_{x}$, (b) $v_{y}$ and (c) $v_{z}$, and the trajectories in the (d) $x-y$ and (e) $y-z$ planes, respectively. Here the initial conditions are $\boldsymbol{r}_{0}=$ $\left(X\left(0.02 y^{*}\right), 0.02 y^{*}, 0\right)$ and $\boldsymbol{v}_{0}=\left(-M_{\mathrm{A}} V_{A}, 0,0\right)$. Note that time is scaled by $2 \pi / \Omega_{x 0 \text { e }}$.

For the proton of large mass, the uncoupled oscillation stops after is has left the diffusion region, and $v_{z}$ still increases linearly with time. Till then, it cannot be accelerated along the meandering orbit to a higher velocity than the Alfvén velocity. The trajectory becomes a cycloid in the $y-z$ plane. It is trapped inside the discontinuities $|x|<X-x^{*}$, since the drift velocity parallel to the $y$-axis $V_{\mathrm{A}}$ becomes much larger than the velocity of the oscillation perpendicular to the $y$-axis, i.e., $\left|v_{y}\right| v_{x} \mid>\mathrm{d} y / \mathrm{d} X \approx 1 / M_{\mathrm{A}}$ for $y \gg y^{*}$.

\section{Some Properties of the Meandering Electrons}

It is interesting to investigate the flight time of charged particles in the diffusion region, which determines the finite electric conductivity necessary for the reconnection at the neutral point in a collisionless plasma. We only have to look into the motion in the $y-z$ plane. The flight time of an electron for $y_{0} \ll y^{*}$ is roughly given by

$$
\tau_{\mathrm{e}} \approx y^{*} / V_{\mathrm{A}}
$$

from Eq. (16), since $v_{z 0} / \Omega_{x 0 \mathrm{e}}, Q / \Omega_{x 0 \mathrm{e}} \approx V_{\mathrm{A}} / \Omega_{x 0_{\mathrm{e}}}=r_{\mathrm{g} x \mathrm{e}} \ll y^{*}$. It is the traverse time of fluid in MHD analyses. The flight time of a proton for $y_{0} \ll y^{*}$ is rougly given by

$$
\tau_{\mathrm{p}} \approx\left(6 m_{\mathrm{p}} / m_{\mathrm{e}}\right)^{1 / 3} y^{*} / V_{\mathrm{A}}=\left(6 m_{\mathrm{p}} / m_{\mathrm{e}}\right)^{1 / 3} \tau_{\mathrm{e}}
$$

from Eq. (17), since $v_{z 0}$ is small enough to be $v_{z 0} / \Omega_{x 0 \mathrm{p}} \ll y^{*}$ and $\sin \left(\Omega_{x 0 \mathrm{p}} \tau_{\mathrm{p}}\right) \approx$ $\Omega_{x 0 \mathrm{p}} \tau_{\mathrm{p}}-\left(\Omega_{x 0 \mathrm{p}} \tau_{\mathrm{p}}\right)^{3} / 6$. A static electric field will be induced by protons that remain in the diffusion region for a time longer than electrons. This electric field is not considered in this paper.

The ratio of the proton-electron currents owing to the meandering particles is roughly given by

$$
I_{\mathrm{p}} / I_{\mathrm{e}} \approx u_{z \mathrm{p}} /\left|u_{z \mathrm{e}}\right| \approx m_{\mathrm{e}} \tau_{\mathrm{p}} / m_{\mathrm{p}} \tau_{\mathrm{e}} \approx\left(6 m_{\mathrm{e}}^{2} / m_{\mathrm{p}}^{2}\right)^{1 / 3} \ll 1,
$$




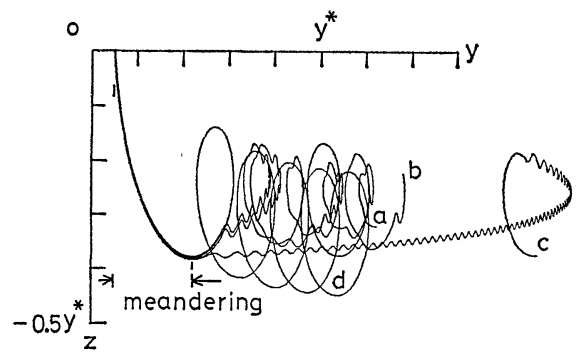

Fig. 5. Electron trajectories in the $y-z$ plane for some different initial perpendicular velocities to the external magnetic field for the case $M_{\mathrm{A}}=0.05$, together with the trajectory obtained from Eq. (15).

where $u_{z}$ is the mean drift velocity near the neutral point. We look into some properties of the meandering electrons which are concentrated in the vicinity of the neutral point.

The meandering motion consists of the drift in the $y-z$ plane leaving the neutral point and the uncoupled oscillation perpendicular to the $y$-axis. Figure 5 shows the electron trajectories in the $y-z$ plane for some different initial perpendicular velocities for the case $M_{\mathrm{A}}=0.05$. The initial conditions are $\boldsymbol{r}_{0}=$ $\left(X\left(0.1 y^{*}\right), 0.1 y^{*}, 0\right)$ and $\boldsymbol{v}_{0}=\left(v_{x 0}, 0,0\right)$ for (a) $v_{x 0}=-M_{\mathrm{A}} V_{\mathrm{A}},(\mathrm{b})-3 M_{\mathrm{A}} V_{\mathrm{A}}$ and (c) $-7 M_{\mathrm{A}} V_{\mathrm{A}}$. The initial perpendicular velocities, for a low- $\beta$ plasma, will have a major effect on the uncoupled oscillation and a minor effect on the motion in the $y-z$ plane. Figure 5 also shows the trajectory (d) obtained from Eq. (15) for the initial conditions $\boldsymbol{r}_{0}=\left(0,0.1 y^{*}, 0\right)$ and $\boldsymbol{v}_{0}=(0,0,0)$. The motion in the $y-z$ plane is well approximated.

If the time, length and velocity are respectively scaled by $1 / \Omega_{x 0}, y^{*}$ and $V_{\mathrm{A}}$, Eqs. (6)-(8) are approximately written as

$$
\begin{gathered}
\mathrm{d} v_{x} / \mathrm{d} t=-v_{z} \Omega_{y} / \Omega_{x 0} \approx-M_{\mathrm{A}}^{-2} v_{z} x, \\
\mathrm{~d} v_{y} / \mathrm{d} t=v_{z} \Omega_{x} / \Omega_{x 0} \approx 2 v_{z} y, \\
\mathrm{~d} v_{z} / \mathrm{d} t=1+v_{x} \Omega_{y} / \Omega_{x 0}-v_{y} \Omega_{x} / \Omega_{x 0} \approx 1+M_{\mathrm{A}}^{-2} v_{x} x-2 v_{y} y,
\end{gathered}
$$

since $|y|<0.5 y^{*}$ and so $\Omega_{x} \approx 2 M_{\mathrm{A}} \Omega_{0} y / y^{*}, \Omega_{y} \approx \Omega_{0} x / x^{*}$ for the meandering electrons. As $M_{\mathrm{A}}$ decreases, the effective spring constant of the uncoupled oscillation becomes stiffer; consequently, the motion in the $y-z$ plane can be better approximated by Eq. (15). From Eqs. $\left(7^{\prime}\right)$ and $\left(8^{\prime}\right)$, it is clear that Eq. (15) does not depend on $M_{\mathrm{A}}$. Thus the motion in the $y-z$ plane for the meandering electrons is well approximated by Eq. (15) for $M_{\mathrm{A}} \lesssim 0.1$, and is almost independent of $M_{\mathrm{A}}$.

Figure 6 shows the $v_{z}-y$ diagram for the meandering electrons obtained from Eq. (15). The mean velocity component $\left|u_{z e}\right|$, though with a considerable dispersion, is two or three times larger than the Alfven velocity outside the diffusion region. For the typical parameters of solar flares, this drift velocity exceeds the electron thermal velocity: $V_{\mathrm{A}}=3.4 \times 10^{6} \mathrm{~m} / \mathrm{sec}$ for $n=10^{17} \mathrm{~m}^{-3}, B=$ $5 \times 10^{-2} \mathrm{~Wb} / \mathrm{m}^{2}$, and $v_{\mathrm{te}}=3.9 \times 10^{6} \mathrm{~m} / \mathrm{sec}$ for $T_{\mathrm{e}}=10^{6}{ }^{\circ} \mathrm{K}$ or $v_{\mathrm{te}}=3.9 \times 10^{5} \mathrm{~m} / \mathrm{sec}$ 


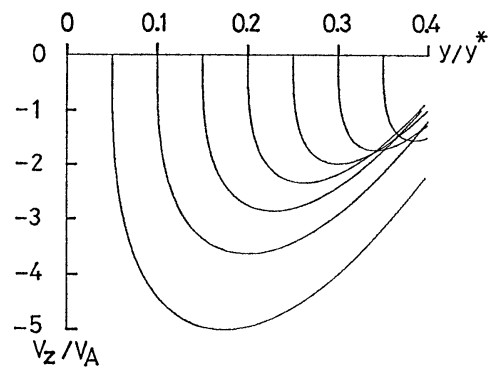

Fig. 6. The $v_{z}-y$ diagram for the meandering electrons obtained from Eq. (15).

if $T_{\mathrm{e}}=10^{4} \mathrm{~K}$, where $v_{\mathrm{te}}$ and $T_{\mathrm{e}}$ are the electron thermal velocity and the temperature, respectively. The current-induced instability by by BunEMAN (1959) may be induced in a small region near the neutral point, which results in a locally high resistivity. For the typical parameters in the magnetotail, this drift velocity cannot exceed the electron thermal velocity but exceeds the phase velocity of the ion-sound wave: $V_{\mathrm{A}}=7.7 \times 10^{5} \mathrm{~m} / \mathrm{sec}$ for $n=5 \times 10^{5} \mathrm{~m}^{-3}, B=2.5 \times$ $10^{-8} \mathrm{~Wb} / \mathrm{m}^{2}$, and $v_{\mathrm{te}}=1.2 \times 10^{7} \mathrm{~m} / \mathrm{sec}$ for $T_{\mathrm{e}}=10^{7} \mathrm{~K}$, on the other hand, $v_{\mathrm{s}}=$ $\left(k T_{\mathrm{e}} / m_{\mathrm{p}}\right)^{1 / 2}=2.9 \times 10^{5} \mathrm{~m} / \mathrm{sec}$ for $T_{\mathrm{e}}=10^{7 \circ} \mathrm{K}$, where $v_{\mathrm{s}}$ and $k$ are the phase velocity of ion-sound wave and the Boltzmann constant, respectively. The similar current-induced instability owing to the ion-sound wave may be induced if $T_{\mathrm{e}} \gg T_{\mathrm{p}}$.

\section{Summary}

The motion of charged particles was studied in a Petschek-type electromagnetic field, on the assumption that the plasma is cold in the external flow region. It is characterized by two gyration frequencies $\Omega_{x}$ and $\Omega_{y}$, usually $\Omega_{x} \ll \Omega_{y}$. They are associated with the motions parallel and perpendicular to the external magnetic field. Outside the diffusion region, the particles predominantly make the $\boldsymbol{E} \times \boldsymbol{B}$ drift. Inside the diffusion region, they make the meandering motion that consists of the drift in the $y-z$ plane starting at the neutral point and the uncoupled oscillation in the perpendicular direction. The mean flight times of the electrons and protons in the diffusion region are given by $\tau_{\mathrm{e}}=y^{*} / V_{\mathrm{A}}$ and $\tau_{\mathrm{p}}=\left(6 m_{\mathrm{p}} / m_{\mathrm{e}}\right)^{1 / 3} \tau_{\mathrm{e}}$, respectively. The meandering electrons are concentrated in the vicinity of the neutral point and have the mean velocity, though with a considerable dispersion, two or three times larger than the Alfvén velocity outside the diffusion region. For the typical parameters of solar flares and in the magnetotail, it is large enough to cause some current-induced instabilities, which result in the high resistivity in a narrow region near the neutral point.

For the typical parameters of solar flares, the above consideration is valid when the electron flight time in the diffusion region is smaller than the classical 
collision time by SPITZER and HÄRM (1953): $\tau_{\mathrm{e}}<\tau_{\mathrm{c}}$, it is written as $M_{\mathrm{A}}>1.6 \times$ $10^{-5}$ for $n=10^{17} \mathrm{~m}^{-3}, B=5 \times 10^{-2} \mathrm{~Wb} / \mathrm{m}^{2}, T=10^{6}{ }^{\circ} \mathrm{K}$ or $M_{\mathrm{A}}>9.5 \times 10^{-3}$ if $T=$ $10^{4} \mathrm{~K}$. For the typical parameters in the magnetotail, the above consideration is valid when the electron flight time in the diffusion region is smaller than that from dusk to dawn sides: $\tau_{\mathrm{e}}<\tau_{\mathrm{d}}$, it is written as $M_{\mathrm{A}}>5.9 \times 10^{-5}$ for $n=5 \times 10^{5}$ $\mathrm{m}^{-3}$, where $\tau_{\mathrm{d}}$ is roughly given by $20 R_{\mathrm{E}} / V_{\mathrm{A}}$ and $R_{\mathrm{E}}$ is the earth radius. Note that $\tau_{\mathrm{c}} \gg \tau_{\mathrm{d}}$ for $n=5 \times 10^{-5} \mathrm{~m} / \mathrm{sec} B=2.5 \times 10^{-8} \mathrm{~Wb} / \mathrm{m}^{2}$ and $T=10^{7 \circ} \mathrm{K}$.

The computer program was tested and run at the Hokkaido University Computing Center. This work was partially funded by the Institute of Plasma Physics, Nagoya University, Nagoya, Japan.

\section{REFERENCES}

Amano, K. and T. Tsuda, Reconnection of magnetic field lines by clounds-in-cells plasma model, J. Geomag. Geoelectr., 29, 9-17, 1977.

Buneman, O., Dissipation of currents in ionized media, Phys. Rev., 115, 503-517, 1959.

DUNGEY, J.W., Conditions for the occurrence of electrical discharges in astrophysical system, Philos. Mag., Ser. 7, 44, 725-738, 1953.

EASTWOOD, J.W., Consistency of fields and particle motion in the 'Speiser' model of the current sheet, Planet. Space Sci., 20, 1555-1568, 1972.

PARKER, E.N., The solar-flare phenomenon and the theory of reconnection and annihilation of magnetic fields, Astrophys. J. Suppl., 8, 177-212, 1963.

Petscher, H.E., Magnetic field annihilation, in AAS-NASA Symposium on the Physics of Solar Flares, edited by W.N. Hess, 425 pp., NASA SP-50, 1964.

SONNERUP, B.U.Ö., Magnetic-field re-connexion in a highly conducting incompressible fluid, $J$. Plasma Phys., 4, 161-174, 1970.

SONNERUP, B.U.Ö., Adiabatic particle orbits in a magnetic null sheet, J. Geophys. Res., 76, 82118222, 1971.

SPeISER, T.W., Particle trajectories in model current sheets, 1. Analytical solutions, J. Geophys. Res., 70, 4219-4226, 1965.

Speiser, T.W., On the uncoupling of parallel and perpendicular particle motion in a neutral sheet, J. Geophys. Res., 73, 1112-1113, 1968.

SPeiser, T.W., Conductivity without collisions or noise, Planet. Space Sci., 18, 613-622, 1970.

Spitzer, L., Jr. and R. HÄrm, Transport phenomena in a completely ionized gas, Phys. Rev., 89, 977-981, 1953.

Vasyliunas, V.M., Theoretical models of magnetic field line merging I, Rev. Geophys. Space Phys., 13, 303-336, 1975.

Yен, T. and W.I. AXFoRD, On the re-connexion of magnetic field lines in conducting fluids, $J$. Plasma Phys., 4, 207-229, 1970. 